\title{
Genetic Variability and Correlation Studies for Yield and Yield Related Traits in Rice (Oryza sativa L.) Genotypes
}

\author{
Kritika Williams*, Aman Mishra, Akansha Verma, BG. Suresh and G. R. Lavanya \\ Department of Genetics and Plant Breeding, Sam Higginbottom University of Agriculture \\ Technology and Sciences, Prayagraj, Uttar Pradesh, India \\ *Corresponding author
}

\section{A B S T R A C T}

\section{Keywords}

Rice (Oryza sativa L.), GCV, PCV, Variability, Heritability, Correlation and Path analysis

\section{Article Info}

\section{Accepted:}

12 December 2020 Available Online: 10 January 2021
The present study consist of 29 rice genotypes including one check variety that were evaluated at Field Experimentation Centre of the Department of Genetics and Plant Breeding, Naini Agricultural Institute, Sam Higginbottom University of Agriculture, Technology and Sciences, Prayagraj U.P. to study genetic variability, heritability, genetic advance, correlation and path coefficient for 13 quantitative characters. The experiment was conducted by using Randomized Block Design with three replications during Kharif 2019. Observation recorded to study the genetic variability parameters, correlation coefficient and path coefficient for yield and its attributing traits. Significant variability reported for all the characters. On the basis of mean performance IRRI-156 was found to be superior in grain yield per hill followed by TP- 30441 and NDR-1045. High to moderate GCV and PCV were recorded for grain yield per hill followed by number of spikelets per panicle, biological yield, test weight and flag leaf length. Grain yield indicated significant positive correlation with days to $50 \%$ flowering, panicle length, number of spikelets per panicle at phenotypic level whereas at genotypic level it showed positive and significant correlation with number of panicle and plant height. Path coefficient analysis showed positive significant direct effects on grain yield per hill were exhibited by plant height and number of panicles at genotypic level whereas it showed positive and significant direct effect for panicle length and number of spikelets per panicle at phenotypic level. Thus, these traits are identified as the efficient and potential for indirect selection for the improvement of rice productivity in the present experimental materials.

\section{Introduction}

Rice (Oryza sativa L.) is one of the staple cereal crops of the world and it is one of the main sources of carbohydrate for nearly one half of the world population. However, more than $90 \%$ of this rice is produced and consumed in Asia, where it is a staple for a majority of the population, including the region's 560 million hungry people. Cultivation of rice is important for the food security of Asia. India has a long history of rice cultivation and stands first in rice area and second in rice production, after China. Rice belongs to the genus Oryza and the tribe Oryzeae of the family Gramineae (Poaceae). 
The genus Oryza contains 25 recognized species, of which 23 are wild species and two, $O$. sativa and $O$. glaberrima are cultivated (Morishima, 1984; Vaughan, 1994; Brar and Khush, 2003). O. sativa is the most widely grown of the two cultivated species. It is grown worldwide including in Asian, North and South American, European Union, Middle Eastern and African countries. However, O. glaberrima is grown solely in West African countries.

The current global population of 7.7 billion is expected to reach 9.7 billion by 2050 (Department of Economics and Social Affairs-2018-19).Globally rice is cultivated now on 166.5 million hectares with annual production if around 760 million tones and average productivity of 2.65 tons/ha (Food and Agriculture Organization, 2018).In India the production of rice was 116.42 Million tons from 43.79 million ha of area and it stands in second position in the world production after china. In Uttar Pradesh area, production and productivity is 5.75 million ha, 15.54 Million tons and $2.70 \mathrm{q} / \mathrm{ha}$ respectively. It is estimated that in India, the demand for rice will be 129.6 million tons by 2040 and 137.3 million tons by 2040 and 137.3 million tons by 2050 for internal consumption. (Directorate of Economics and Statistics, 2018-19).

As per the present scenario, the population of our country continuously increasing at an alarming pace, so there is a need for identification and releasing of the promising germplams having higher yield and would be able to serve as a source of nutritional security in the near future.

\section{Materials and Methods}

The experimental material consist of 29 rice genotypes which were obtained from department of Genetics and Plant Breeding,
Naini Agricultural Institute, Sam Higginbottom University of Agriculture and Sciences, Prayagraj were grown during Kharif season of 2018 at Field Experimentation Centre, SHUATS, Prayagraj (U.P), India. These genotypes were planted in Randomized Block Design with three replications. Thirty days old seedlings were transplanted in the experimental site with the spacing of $15 \mathrm{~cm}$ between plants and $20 \mathrm{~cm}$ between the rows, keeping single seedling per hill. Fertilizer dose of $120 \mathrm{~kg} \mathrm{~N}, 60 \mathrm{~kg} \mathrm{P}_{2} \mathrm{O}_{5}$ and $40 \mathrm{~kg} \mathrm{~K}_{2} \mathrm{O}$ was applied. The standard agronomic practices were adopted for normal crop growth.

Thirteen quantitative characters were used to characterize and assess the genetic variability, heritability, genetic advance, correlation coefficient and path analysis. Five plants were randomly selected from each replication in each genotype for the analysis of menetion parameters. The observations were recorded as per the standard procedure and subjected to statistical analysis using mean values of five randomly selected plants.

\section{Statistical analysis}

The data on quantitative characters were statistically analysed on the basis of model described by Panse and Sukhatme (1967) for randomized block design. Parameters of genetic variability include mean, range, genotypic and phenotypic coefficient of variation were calculated by the method suggested by Burton and De Vane (1953) and th estimate of PCV and GCV were classified as low, moderate and high according to Sivasubramanian and Madhavamenon (1973).

Heritability for broad sense is the ratio of genotypic variance to the total phenotypic variance. Expected genetic advance was calculated by the method suggested by Burton and De Vane (1955) and genetic advance as 
percentage of mean was calculated by the following formula:

$$
\text { GA } \% \mathrm{M}=\quad-\begin{gathered}
\mathrm{GA} \\
\mathrm{X}^{-}
\end{gathered} \quad \mathrm{x} 100
$$

Correlation coefficients were calculated for all quantitative character combinations at phenotypic and genotypic level by the formula given by Johnson et al., (1958).

Genotypic correlation between traits $\mathrm{X}$ and $\mathrm{Y}$ :

$$
R_{x y}(g)=\frac{\sigma_{g}^{2}(x y)}{\sqrt{\sigma_{g}^{2}{ }^{2} \sigma_{g}^{2}}}
$$

Phenotypic correlation between traits $\mathrm{X}$ and Y:

$R_{x y}(p)=\frac{\sigma_{p}^{2}(x y)}{\sqrt{\sigma_{p}^{2} x \sigma_{p}^{2}}}$

The direct and indirect contribution of various characters to yield were calculated through path coefficient analysis as suggested by Wright (1921) and elaborated by Dewey and $\mathrm{Lu}$ (1959). The path coefficients were rated based on the scales given below

$>1.00$ - Very High

0.3-0.99 - High

0.2-0.29 - Moderate

0.1-0.19 - Low

0.0-0.09 - Negligible

\section{Results and Discussion}

The analysis of variance exhibited the presence of significant differences among the tested genotypes for all the characters indicating the existence of variability. The analysis of variance showed that the genotypes differed significantly $(\mathrm{p}<0.05)$ for all the studied characters. The presence of large amount of variability might be due to diverse source of materials taken as well as environmental influence affecting the phenotypes. Kumar and Saravanan et al., (2012); Osman et al., (2014) and Fentie et al., (2014) reported significance differences among rice genotypes evaluated in different locations.

\section{Genetic variability}

The magnitude of variation between was reflected by high values of mean and range for the traits studied (Table 2). The value of GCV and PCV were obtained high for number of spikelets per panicle, number of tillers and number of panicles. This was in agreement with the finding reported by Roy et al (2017), Kishore et al (2018) and Sandeep et al (2018). In this study moderate value of PCV and GCV were recorded in test weight, flag leaf width, flag leaf length and harvest index.

These results are in accordance with Dhurai et al (2014) and Rashid et al (2019). Low magnitude of genotypic and phenotypic coefficient of variation for days to maturity, days to $50 \%$ flowering, plant height and panicle length was observed. Difference between the magnitude of GCV and PCV was very low for these characters indicate the role of genotypic in expression of these characters.

\section{Heritability and genetic advance}

In our study, The estimates of heritability coupled with high genetic advance as percent of mean was observed for number of spikelets per panicle, biological yield flag leaf length, flag leaf width, harvest index, test weight and grain yield suggesting the scope of selection for improvement of these characters. The results in respect to heritability and genetic advance are in agreement with earlier reports on rice by Meena et al., (2016), Nandan et al., (2018), Nuruzzaman et al., (2017), Lingaiah et al., (2019), Barik et al., (2020). 
Table.1 Analysis of variance for 13 different quantitative characters in rice genotypes

\begin{tabular}{|c|c|c|c|}
\hline \multirow{2}{*}{ Characters } & \multicolumn{3}{|c|}{ Mean Sum of Squares } \\
\cline { 2 - 4 } & Replications & Treatments & Error \\
\cline { 2 - 4 } & $(\mathbf{d f =})$ & $\mathbf{( d f = 2 8 )}$ & $\mathbf{( d f = 5 6 )}$ \\
\hline Days to 50\% Flowering & 6.85 & $85.94^{* *}$ & 5.80 \\
\hline Plant Height & 82.43 & $218.99^{* *}$ & 24.98 \\
\hline Number of Tillers/ Plant & 1.66 & $8.87^{* *}$ & 2.54 \\
\hline Number of Panicles/ Plant & 1.78 & $8.80^{* *}$ & 1.69 \\
\hline Panicle Length & 1.73 & $6.96^{* *}$ & 1.17 \\
\hline Flag Leaf Length & 2.60 & $52.11^{* *}$ & 8.43 \\
\hline Flag Leaf Width & 0.00026 & $0.072^{* *}$ & 0.009 \\
\hline Number of spikelets per Panicle & 26.51 & $3818.34 * *$ & 6.59 \\
\hline Days to Maturity & 0.87 & $102.52^{* *}$ & 1.17 \\
\hline Biological Yield & 8.52 & $556.66^{* *}$ & 10.22 \\
\hline Harvest Index & 30.42 & $49.82^{* *}$ & 6.26 \\
\hline Test Weight & 1.10 & $24.36^{* *}$ & 2.20 \\
\hline Grain Yield/Plant & 10.59 & $148.55^{* *}$ & 4.19 \\
\hline
\end{tabular}

$* * 1 \%$ level of significance

Table.2 Estimation of Genetic parameters for 13 quantitative characters in rice genotypes

\begin{tabular}{|c|c|c|c|c|c|c|c|c|}
\hline S. No. & Characters & $\boldsymbol{\sigma}^{\mathbf{2}}$ & $\boldsymbol{\sigma}^{\mathbf{2}} \mathbf{p}$ & $\mathbf{G C V}$ & $\mathbf{P C V}$ & $\mathbf{h}^{\mathbf{2}}(\boldsymbol{\%})$ & $\mathbf{G A}$ & $\mathbf{G A} \boldsymbol{\%} \mathbf{M e a n}$ \\
\hline $\mathbf{1 .}$ & $\begin{array}{c}\text { Days to 50\% } \\
\text { Flowering }\end{array}$ & 26.71 & 32.57 & 5.51 & 6.08 & 82.16 & 9.65 & 10.29 \\
\hline $\mathbf{2 .}$ & Plant Height & 64.67 & 89.65 & 6.72 & 7.91 & 72.13 & 14.07 & 11.76 \\
\hline $\mathbf{3 .}$ & Tillers per Hill & 2.11 & 4.65 & 13.95 & 20.70 & 45.38 & 2.01 & 19.35 \\
\hline $\mathbf{4 .}$ & Panicles per Hill & 2.37 & 4.06 & 15.72 & 20.60 & 58.30 & 2.42 & 24.74 \\
\hline $\mathbf{5 .}$ & Panicle Length & 1.93 & 3.10 & 5.23 & 6.63 & 62.18 & 2.25 & 8.49 \\
\hline $\mathbf{6 .}$ & Flag Leaf Length & 14.56 & 22.99 & 10.13 & 12.73 & 63.31 & 6.25 & 16.61 \\
\hline $\mathbf{7 .}$ & Flag Leaf Width & 0.02 & 0.03 & 9.88 & 11.86 & 69.43 & 0.24 & 16.96 \\
\hline $\mathbf{8 .}$ & Spikelet's per & 1270.58 & 1271.71 & 18.36 & 18.41 & 99.48 & 73.23 & 37.73 \\
\hline $\mathbf{9 .}$ & Panicle & & & & & & & \\
\hline $\mathbf{1 0 .}$ & Days to Maturity & 33.78 & 34.95 & 4.71 & 4.79 & 96.65 & 11.71 & 9.54 \\
\hline $\mathbf{1 1 .}$ & Hiological Yield & 182.14 & 192.37 & 15.46 & 15.89 & 94.68 & 27.05 & 31.00 \\
\hline $\mathbf{1 2}$ & Harvest Index & 14.52 & 20.78 & 9.15 & 10.95 & 69.87 & 6.56 & 15.77 \\
\hline $\mathbf{1 3 .}$ & Gest Weight & 7.38 & 9.60 & 10.74 & 12.25 & 76.87 & 4.90 & 19.41 \\
\hline
\end{tabular}

$\sigma^{2} \mathrm{~g}=$ Genotypic variance. $\quad \sigma^{2} \mathrm{p}=$ Phenotypic variance. $\quad \mathrm{H}^{2}=$ Heritability

$\mathrm{GCV}=$ Genotypic coefficient of variation.

$\mathrm{PCV}=$ Phenotypic coefficient of variation 
Table.3 Correlation coefficient between yield and its components characters in rice genotypes at phenotypic level

\begin{tabular}{|c|c|c|c|c|c|c|c|c|c|c|c|c|c|}
\hline Character & $\begin{array}{c}\text { Days to } \\
50 \% \\
\text { Flowering }\end{array}$ & $\begin{array}{c}\text { Plant } \\
\text { Height }\end{array}$ & $\begin{array}{c}\text { No of } \\
\text { Tillers/ } \\
\text { plant }\end{array}$ & $\begin{array}{c}\text { No of } \\
\text { Panicles/ } \\
\text { Plant }\end{array}$ & $\begin{array}{l}\text { Panicle } \\
\text { Length }\end{array}$ & $\begin{array}{c}\text { Flag } \\
\text { Leaf } \\
\text { Length }\end{array}$ & $\begin{array}{c}\text { Flag } \\
\text { Leaf } \\
\text { Width }\end{array}$ & $\begin{array}{c}\text { No of } \\
\text { spikelets } \\
\text { per } \\
\text { Panicle }\end{array}$ & $\begin{array}{c}\text { Days to } \\
\text { maturity }\end{array}$ & $\begin{array}{l}\text { Biological } \\
\text { yield }\end{array}$ & $\begin{array}{c}\text { Harvest } \\
\text { Index }\end{array}$ & $\begin{array}{c}\text { Test } \\
\text { weight }\end{array}$ & $\begin{array}{c}\text { Grain Yield/ } \\
\text { Plant }\end{array}$ \\
\hline $\begin{array}{c}\text { Days to } 50 \% \\
\text { Flowering }\end{array}$ & 1.00 & -0.10 & -0.009 & 0.003 & $0.30 * *$ & -0.15 & $0.21 *$ & $0.31 * *$ & $0.79 * * *$ & $0.29 * *$ & 0.80 & 0.01 & $0.278 *$ \\
\hline Plant Height & & 1.00 & 0.05 & 0.59 & 0.21 & $0.44 * *$ & $0.46^{* *}$ & 0.70 & -0.07 & $0.22 *$ & 0.008 & 0.065 & 0.186 \\
\hline $\begin{array}{c}\text { No of Tillers/ } \\
\text { plant }\end{array}$ & & & 1.00 & $0.92 * *$ & 0.20 & 0.10 & 0.02 & 0.15 & 0.41 & 0.03 & $0.24 *$ & -0.13 & 0.140 \\
\hline $\begin{array}{c}\text { No of } \\
\text { Panicles/ } \\
\text { Plant }\end{array}$ & & & & 1.00 & 0.10 & 0.07 & -0.01 & 0.20 & 0.08 & 0.08 & $0.27 *$ & -0.17 & 0.187 \\
\hline $\begin{array}{c}\text { Panicle } \\
\text { Length }(\mathrm{cm})\end{array}$ & & & & & 1.00 & 0.14 & $0.23 *$ & 0.207 & 0.202 & 0.209 & 0.208 & -.88 & $0.284 * *$ \\
\hline $\begin{array}{l}\text { Flag Leaf } \\
\text { Length }\end{array}$ & & & & & & 1.00 & $0.46^{* *}$ & 0.14 & -0.03 & -0.07 & -0.14 & -0.63 & -0.127 \\
\hline $\begin{array}{l}\text { Flag Leaf } \\
\text { Width }\end{array}$ & & & & & & & 1.00 & 0.20 & $0.34 * *$ & 0.03 & -0.14 & $-0.21 *$ & -0.106 \\
\hline $\begin{array}{c}\text { No of spikelets } \\
\text { per Panicle }\end{array}$ & & & & & & & & 1.00 & $0.33 * *$ & $0.38 * *$ & 0.03 & $-0.21 *$ & $0.332 * *$ \\
\hline $\begin{array}{l}\text { Days to } \\
\text { maturity }\end{array}$ & & & & & & & & & 1.00 & 0.14 & 0.02 & -0.14 & 0.009 \\
\hline $\begin{array}{l}\text { Biological } \\
\text { yield }\end{array}$ & & & & & & & & & & 1.00 & 0.11 & 0.10 & 0.855 \\
\hline Harvest Index & & & & & & & & & & & 1.00 & 0.13 & 0.603 \\
\hline Test weight & & & & & & & & & & & & 1.00 & 0.175 \\
\hline \begin{tabular}{cc}
\multicolumn{2}{c}{ Grain } \\
Yield/ Plant
\end{tabular} & & & & & & & & & & & & & 1.00 \\
\hline
\end{tabular}


Table.4 Correlation coefficient between yield and its components characters in rice genotypes at genotypic level

\begin{tabular}{|c|c|c|c|c|c|c|c|c|c|c|c|c|c|}
\hline Character & $\begin{array}{c}\text { Days to } \\
50 \% \\
\text { Flowering }\end{array}$ & $\begin{array}{c}\text { Plant } \\
\text { Height }\end{array}$ & $\begin{array}{c}\text { No of } \\
\text { Tillers/ } \\
\text { plant }\end{array}$ & $\begin{array}{c}\text { No of } \\
\text { Panicles/ } \\
\text { Plant }\end{array}$ & $\begin{array}{l}\text { Panicle } \\
\text { Length }\end{array}$ & $\begin{array}{c}\text { Flag } \\
\text { Leaf } \\
\text { Length }\end{array}$ & $\begin{array}{c}\text { Flag } \\
\text { Leaf } \\
\text { Width }\end{array}$ & $\begin{array}{c}\text { No of } \\
\text { spikelets } \\
\text { per } \\
\text { Panicle }\end{array}$ & $\begin{array}{c}\text { Days to } \\
\text { maturity }\end{array}$ & $\begin{array}{l}\text { Biological } \\
\text { yield }\end{array}$ & $\begin{array}{c}\text { Harvest } \\
\text { Index }\end{array}$ & $\begin{array}{c}\text { Test } \\
\text { weight }\end{array}$ & $\begin{array}{l}\text { Grain } \\
\text { Yield/ } \\
\text { Plant }\end{array}$ \\
\hline $\begin{array}{c}\text { Days to } 50 \% \\
\text { Flowering }\end{array}$ & 1.00 & -0.182 & -0.016 & -0.031 & 0.424 & -0.172 & $0.309 * *$ & 0.351 & 0.889 & $0.337 * *$ & 0.164 & 0.017 & 0.348 \\
\hline Plant Height & & 1.00 & 0.195 & 0.186 & 0.136 & 0.615 & 0.536 & 0.084 & -0.081 & $0.282 * *$ & 0.025 & 0.149 & $0.242 *$ \\
\hline $\begin{array}{l}\text { No of Tillers/ } \\
\text { plant }\end{array}$ & & & 1.00 & 0.998 & 0.148 & $0.293 * *$ & 0.178 & $0.228 *$ & 0.046 & -0.008 & 0.452 & $-0.214^{*}$ & 0.188 \\
\hline $\begin{array}{c}\text { No of Panicles/ } \\
\text { Plant }\end{array}$ & & & & 1.00 & $0.264 *$ & $0.222 *$ & $0.215^{*}$ & $0.265^{*}$ & 0.090 & 0.080 & 0.478 & $-0.246^{*}$ & $0.271 *$ \\
\hline $\begin{array}{l}\text { Panicle Length } \\
(\mathrm{cm})\end{array}$ & & & & & 1.00 & 0.13 & 0.146 & $0.275^{* *}$ & $0.255^{*}$ & $0.304 * *$ & 0.426 & 0.238 & 0.445 \\
\hline Flag Leaf Length & & & & & & 1.00 & 0.563 & 0.180 & -0.056 & -0.100 & $-0.211 *$ & -0.081 & -0.165 \\
\hline Flag Leaf Width & & & & & & & 1.00 & $0.247 *$ & 0.412 & -0.033 & -0.194 & $-0.234 *$ & -0.119 \\
\hline $\begin{array}{c}\text { No of spikelets } \\
\text { per Panicle }\end{array}$ & & & & & & & & 1.00 & $0.342 * *$ & 0.397 & 0.037 & $-0.242 *$ & -0.347 \\
\hline Days to maturity & & & & & & & & & 1.00 & 0.148 & -0.025 & -0.134 & 0.103 \\
\hline Biological yield & & & & & & & & & & 1.00 & 0.171 & 0.119 & 0.886 \\
\hline Harvest Index & & & & & & & & & & & 1.00 & -0.122 & 0.602 \\
\hline Test weight & & & & & & & & & & & & 1.00 & 0.170 \\
\hline $\begin{array}{c}\text { Grain Yield/ } \\
\text { Plant }\end{array}$ & & & & & & & & & & & & & 1.00 \\
\hline
\end{tabular}


Table.5 Estimation of path coefficient at phenotypic level for 13 quantitative characters with grain yield in rice genotypes

\begin{tabular}{|c|c|c|c|c|c|c|c|c|c|c|c|c|c|}
\hline Character & $\begin{array}{c}\text { Days to } \\
50 \% \\
\text { Flowering }\end{array}$ & $\begin{array}{c}\text { Plant } \\
\text { Height }\end{array}$ & $\begin{array}{c}\text { No of } \\
\text { Tillers/ } \\
\text { plant }\end{array}$ & $\begin{array}{c}\text { No of } \\
\text { Panicles/ } \\
\text { Plant }\end{array}$ & $\begin{array}{l}\text { Panicle } \\
\text { Length }\end{array}$ & $\begin{array}{l}\text { Flag } \\
\text { Leaf } \\
\text { Length }\end{array}$ & $\begin{array}{c}\text { Flag } \\
\text { Leaf } \\
\text { Width }\end{array}$ & $\begin{array}{c}\text { No of } \\
\text { spikelets } \\
\text { per } \\
\text { Panicle }\end{array}$ & $\begin{array}{l}\text { Days to } \\
\text { maturity }\end{array}$ & $\begin{array}{c}\text { Biological } \\
\text { yield }\end{array}$ & $\begin{array}{c}\text { Harvest } \\
\text { Index }\end{array}$ & $\begin{array}{c}\text { Test } \\
\text { weight }\end{array}$ & $\begin{array}{l}\text { Grain } \\
\text { Yield/ } \\
\text { Plant }\end{array}$ \\
\hline $\begin{array}{c}\text { Days to } 50 \% \\
\text { Flowering }\end{array}$ & -0.028 & 0.005 & 0.0005 & -0.0009 & -0.012 & 0.004 & -0.008 & -0.009 & -0.025 & -0.009 & -0.0004 & -0.0005 & 0.348 \\
\hline Plant Height & 0.006 & -0.033 & -0.006 & -0.006 & -0.004 & -0.020 & -0.017 & -0.002 & 0.002 & -0.009 & -0.0009 & -0.005 & 0.246 \\
\hline $\begin{array}{l}\text { No of Tillers/ } \\
\text { plant }\end{array}$ & -0.0007 & 0.008 & 0.041 & 0.041 & 0.006 & 0.012 & 0.007 & 0.009 & 0.002 & -0.003 & 0.018 & -0.009 & 0.188 \\
\hline $\begin{array}{c}\text { No of } \\
\text { Panicles/ } \\
\text { Plant }\end{array}$ & -0.002 & -0.014 & -0.075 & -0.075 & -0.020 & -0.016 & -0.016 & -0.020 & -0.006 & -0.006 & -0.036 & 0.018 & 0.271 \\
\hline $\begin{array}{l}\text { Panicle } \\
\text { Length }\end{array}$ & -0.002 & -0.0008 & -0.0009 & -0.001 & -0.005 & -0.008 & -0.008 & -0.001 & -0.001 & -0.001 & -0.002 & -0.001 & 0.445 \\
\hline $\begin{array}{l}\text { Flag Leaf } \\
\text { Length }\end{array}$ & -0.005 & 0.020 & 0.009 & 0.007 & 0.004 & 0.032 & 0.018 & 0.005 & -0.001 & -0.003 & -0.006 & -0.002 & - \\
\hline $\begin{array}{l}\text { Flag Leaf } \\
\text { Width }\end{array}$ & 0.005 & 0.009 & 0.003 & 0.003 & 0.002 & 0.09 & 0.017 & 0.004 & 0.007 & -0.006 & -0.003 & -0.004 & $\begin{array}{l}- \\
0.119\end{array}$ \\
\hline $\begin{array}{l}\text { No of } \\
\text { spikelets per } \\
\text { Panicle }\end{array}$ & 0.006 & 0.001 & 0.004 & 0.004 & 0.004 & 0.003 & 0.004 & 0.018 & 0.006 & 0.007 & 0.0007 & -0.004 & 0.347 \\
\hline $\begin{array}{l}\text { Days to } \\
\text { maturity }\end{array}$ & 0.012 & -0.001 & 0.0006 & 0.001 & 0.003 & -0.008 & 0.005 & 0.004 & 0.013 & 0.002 & -0.0004 & -0.001 & 0.103 \\
\hline $\begin{array}{c}\text { Biological } \\
\text { yield }\end{array}$ & 0.278 & 0.231 & -0.0068 & 0.066 & 0.249 & -0.082 & -0.027 & 0.326 & 0.122 & 0.821 & 0.140 & 0.097 & 0.886 \\
\hline $\begin{array}{l}\text { Harvest } \\
\text { Index }\end{array}$ & 0.081 & 0.012 & 0.223 & 0.236 & 0.210 & -0.10 & -0.096 & 0.018 & -0.012 & 0.084 & 0.494 & 0.060 & 0.602 \\
\hline Test weight & 0.0004 & 0.003 & -0.004 & -0.005 & 0.005 & -0.001 & -0.005 & -0.005 & -0.003 & 0.002 & 0.002 & 0.022 & 0.170 \\
\hline
\end{tabular}


Table.6 Estimation of path coefficient at genotypic level for 13 quantitative characters with grain yield in rice genotypes

\begin{tabular}{|c|c|c|c|c|c|c|c|c|c|c|c|c|c|}
\hline Character & $\begin{array}{c}\text { Days to } \\
50 \% \\
\text { Flowering }\end{array}$ & $\begin{array}{c}\text { Plant } \\
\text { Height }\end{array}$ & $\begin{array}{c}\text { No of } \\
\text { Tillers/ } \\
\text { plant }\end{array}$ & $\begin{array}{c}\text { No of } \\
\text { Panicles/ } \\
\text { Plant }\end{array}$ & $\begin{array}{l}\text { Panicle } \\
\text { Length }\end{array}$ & $\begin{array}{l}\text { Flag } \\
\text { Leaf } \\
\text { Length }\end{array}$ & $\begin{array}{c}\text { Flag } \\
\text { Leaf } \\
\text { Width }\end{array}$ & $\begin{array}{c}\text { No of } \\
\text { spikelets } \\
\text { per } \\
\text { Panicle }\end{array}$ & $\begin{array}{l}\text { Days to } \\
\text { maturity }\end{array}$ & $\begin{array}{l}\text { Biological } \\
\text { yield }\end{array}$ & $\begin{array}{l}\text { Harvest } \\
\text { Index }\end{array}$ & $\begin{array}{c}\text { Test } \\
\text { weight }\end{array}$ & $\begin{array}{l}\text { Grain } \\
\text { Yield/ } \\
\text { Plant }\end{array}$ \\
\hline $\begin{array}{c}\text { Days to } 50 \% \\
\text { Flowering }\end{array}$ & -0.028 & 0.005 & 0.0005 & -0.0009 & -0.012 & 0.004 & -0.008 & -0.009 & -0.025 & -0.009 & -0.0004 & -0.0005 & 0.348 \\
\hline Plant Height & 0.006 & -0.033 & -0.006 & -0.006 & -0.004 & -0.020 & -0.017 & -0.002 & 0.002 & -0.009 & -0.0009 & -0.005 & 0.246 \\
\hline $\begin{array}{l}\text { No of Tillers/ } \\
\text { plant }\end{array}$ & -0.0007 & 0.008 & 0.041 & 0.041 & 0.006 & 0.012 & 0.007 & 0.009 & 0.002 & -0.003 & 0.018 & -0.009 & 0.188 \\
\hline $\begin{array}{l}\text { No of } \\
\text { Panicles/ } \\
\text { Plant }\end{array}$ & -0.002 & -0.014 & -0.075 & -0.075 & -0.020 & -0.016 & -0.016 & -0.020 & -0.006 & -0.006 & -0.036 & 0.018 & 0.271 \\
\hline $\begin{array}{l}\text { Panicle } \\
\text { Length }\end{array}$ & -0.002 & -0.0008 & -0.0009 & -0.001 & -0.005 & -0.008 & -0.008 & -0.001 & -0.001 & -0.001 & -0.002 & -0.001 & 0.445 \\
\hline $\begin{array}{l}\text { Flag Leaf } \\
\text { Length }\end{array}$ & -0.005 & 0.020 & 0.009 & 0.007 & 0.004 & 0.032 & 0.018 & 0.005 & -0.001 & -0.003 & -0.006 & -0.002 & $-\overline{0.165}$ \\
\hline $\begin{array}{l}\text { Flag Leaf } \\
\text { Width }\end{array}$ & 0.005 & 0.009 & 0.003 & 0.003 & 0.002 & 0.09 & 0.017 & 0.004 & 0.007 & -0.006 & -0.003 & -0.004 & $-\overline{0.119}$ \\
\hline $\begin{array}{c}\text { No of } \\
\text { spikelets per } \\
\text { Panicle }\end{array}$ & 0.006 & 0.001 & 0.004 & 0.004 & 0.004 & 0.003 & 0.004 & 0.018 & 0.006 & 0.007 & 0.0007 & -0.004 & 0.347 \\
\hline $\begin{array}{l}\text { Days to } \\
\text { maturity }\end{array}$ & 0.012 & -0.001 & 0.0006 & 0.001 & 0.003 & -0.008 & 0.005 & 0.004 & 0.013 & 0.002 & -0.0004 & -0.001 & 0.103 \\
\hline $\begin{array}{l}\text { Biological } \\
\text { yield }\end{array}$ & 0.278 & 0.231 & -0.0068 & 0.066 & 0.249 & -0.082 & -0.027 & 0.326 & 0.122 & 0.821 & 0.140 & 0.097 & 0.886 \\
\hline $\begin{array}{l}\text { Harvest } \\
\text { Index }\end{array}$ & 0.081 & 0.012 & 0.223 & 0.236 & 0.210 & -0.10 & -0.096 & 0.018 & -0.012 & 0.084 & 0.494 & 0.060 & 0.602 \\
\hline Test weight & 0.0004 & 0.003 & -0.004 & -0.005 & 0.005 & -0.001 & -0.005 & -0.005 & -0.003 & 0.002 & 0.002 & 0.022 & 0.170 \\
\hline
\end{tabular}


Fig.1 Histogram depicting GCV \& PCV for 13 quantitative characters of rice genotypes

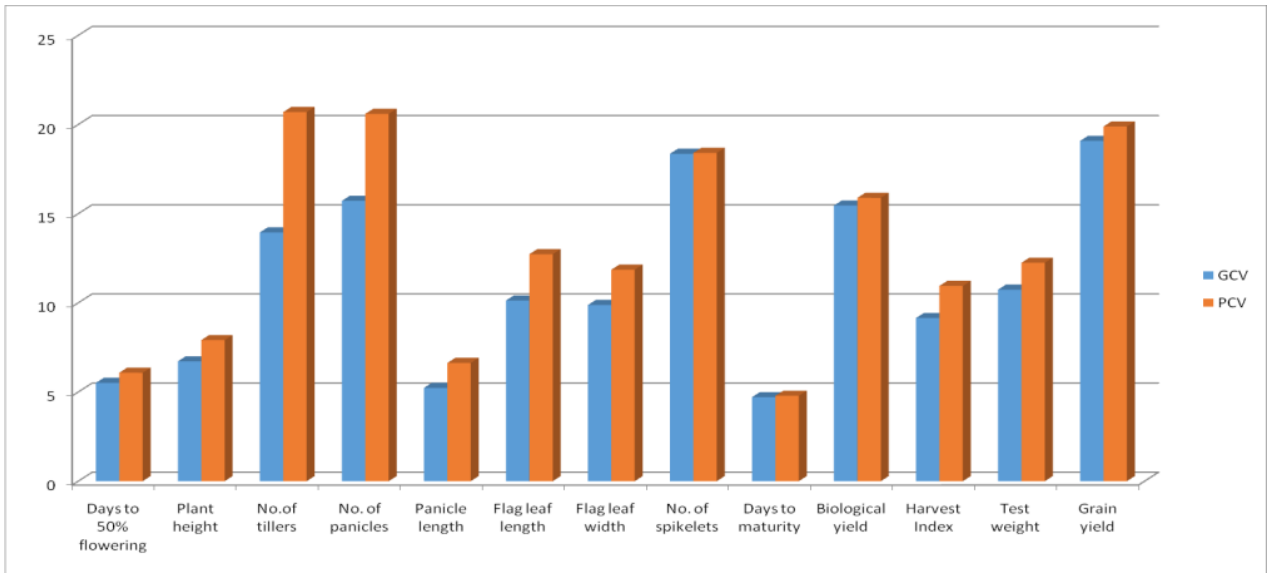

Fig.4.2 Histogram depicting Heritability and Genetic advance for 13 quantitative characters of rice genotypes

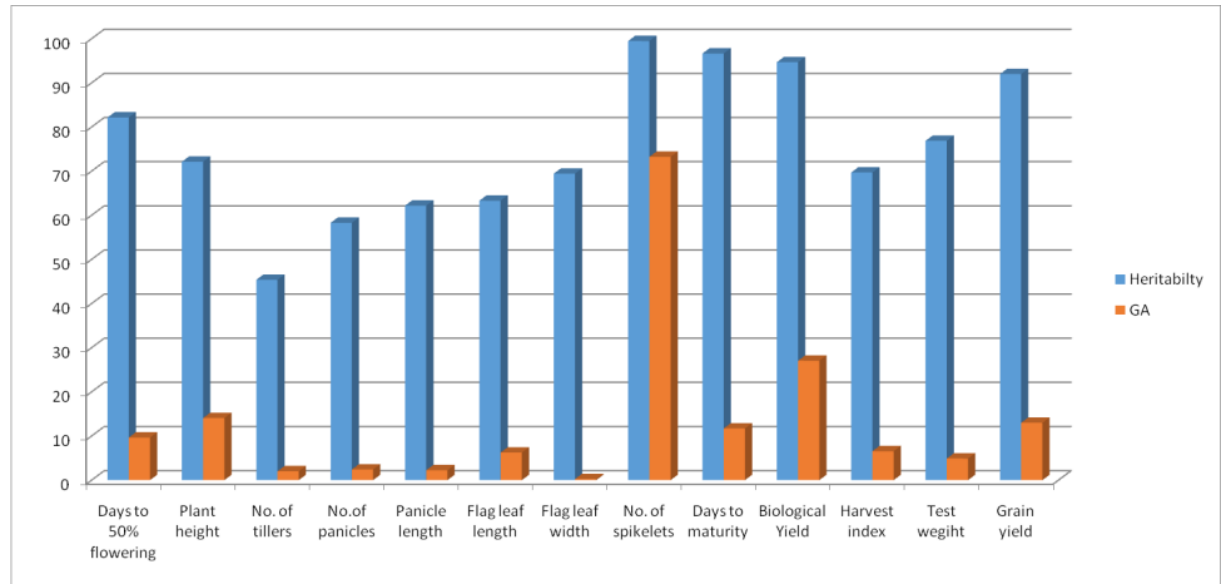

Fig.3 Phenotypic path diagram for grain yield per plant for 13 quantitative characters

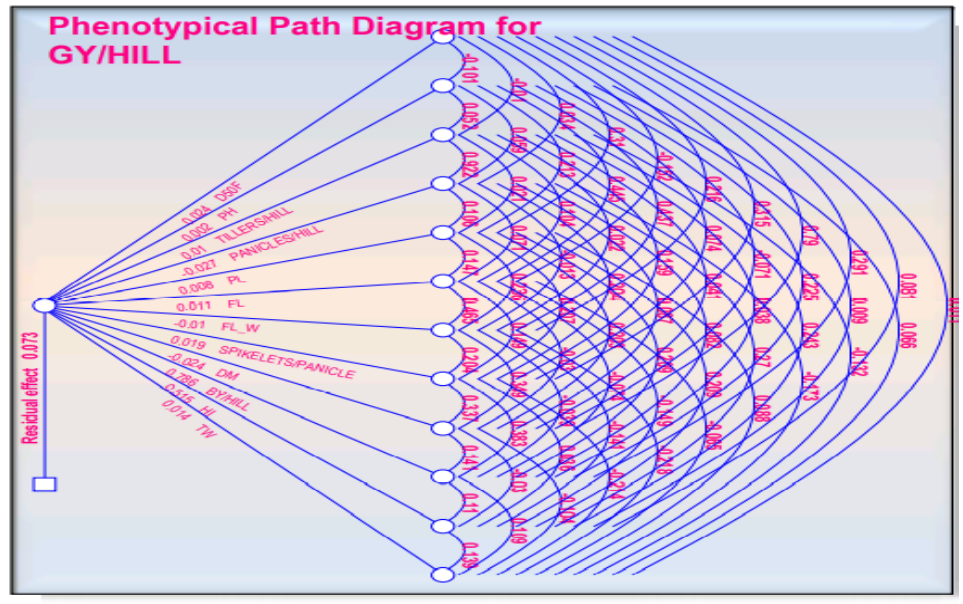


Fig.4 Genotypic path diagram for grain yield per plant for 12 quantitative characters

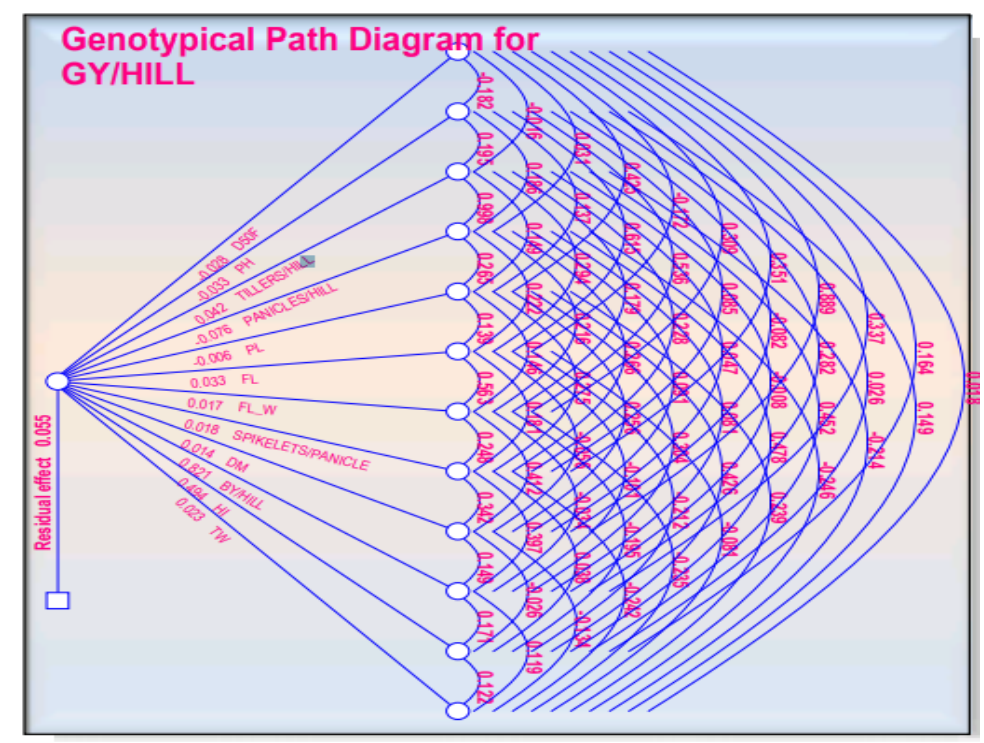

High estimates of Heritability coupled with moderate to low values of Genetic advance as per cent mean were observed for number of spikelets per panicle followed by days to maturity, biological yield, grain yield per hill and days to $50 \%$ flowering suggesting that there was preponderance of additive gene action for the expression of these characters.

\section{Correlation coefficient analysis}

In table $3 \& 4$ it is revealed that genotypic correlation coefficients were higher in magnitude than phenotypic correlation coefficients in the same direction and magnitude indicated that there is a strong inherent association between each pair of characters which might be due to masking or modifying effect of the environment. Correlation coefficient analysis at genotypic level revealed that plant height followed by number of panicles showed positive and significant correlation with grain yield per hill. The negative correlation with grain yield was reported for flag leaf length followed by flag leaf width. Correlation coefficient analysis at phenotypic level revealed that panicle length followed by days to $50 \%$ flowering and number of spikelets per panicle showed positive and significant correlation with grain yield per hill. The negative and non significant correlation was reported for flag leaf length followed by flag leaf width. In the present investigation the related findings were carried out by Singh et al., (2018), Srikanth et al., (2017), Srujana et al., (2017), Srilakshmi et al., (2018), Tefera et al., (2017), Vijaya et al., (2016), Vincent et al., (2017), Rashid et al., (2017), Roy et al., (2015) and Vinoth et al., (2016).

\section{Path coefficient analysis}

In table $5 \& 6$ path coefficient revealed that the highest positive direct effect towards grain yield/plant was observed for plant height followed by number of panicles showed negative significant direct effect on grain yield per plant whereas biological yield, harvest index, number of tillers, flag leaf length, flag leaf width, day to maturity, harvest index and test weight at genotypic level. Path analysis at phenotypic level indicated that number of spikelets per panicle followed by panicle length showed positive significant direct effect on grain yield per hill 
whereas days to $50 \%$ flowering followed by plant height, number of tillers, flag leaf length, biological yield, harvest index and test weight showed positive direct effect on grain yield per hill. From the path analysis these characters may serve as effective selection parameters in direct breeding program for yield improvement in rice.

It is concluded that among 29 genotypes of rice on the basis of mean performance IRRI156 was found to be superior in grain yield per hill over the check variety followed by TP- 30441 and NDR-1045 showed higher yield over the check. In days to $50 \%$ flowering OM-6070 is the earliest and CT18148-6-9-5-1-2MMP is recorded as longest duration for days to $50 \%$ flowering for genotypes studied. Based on the mean performance, plant height is high for TP30439 and low for CT-18148-6-9-S-4.For Days to maturity OM-6070 is the earliest and CT-18148-6-9-5-1-2MMP is the longest duration of days to maturity. For test weight the highest test weight is recorded for IRRI181 and the lowest test weight test weight is depicted by IRRI-123 for genotypes studied. High to moderate estimates of GCV and PCV were recorded for grain yield per hill followed by number of spikelets per panicle, biological yield, test weight and flag leaf length. High estimates of Heritability coupled with moderate to low values of Genetic advance as per cent mean was observed for number of spikelets per panicle followed by days to maturity, biological yield, grain yield per hill and days to $50 \%$ flowering suggesting that there was preponderance of additive gene action for the expression of these characters. Grain yield indicated significant positive correlation with days to $50 \%$ flowering followed by panicle length, number of spikelets per panicle in terms of phenotypic correlation coefficient whereas in terms of genotypic coefficient grain yield per hill showed positive and significant correlation with number of panicle and plant height. Path coefficient analysis showed positive significant direct effects on grain yield per hill were exhibited by plant height and number of panicles at genotypic level whereas it showed positive and significant direct effect for panicle length and number of spikelets per panicle at phenotypic level. Therefore, they seem to be primary yield contributing character and thus can be used as direct selection to improve genetic yield potential of rice.

\section{References}

Annual Report, Department of Agriculture, Co-operation \& Farmers Welfare (2018-19).

Abebe T, Alamerew S, Tulu L (2017).Genetic Variability, Heritability and Genetic Advance for Yield and its Related Traits in Rainfed Lowland Rice (Oryza sativa L.). Advances in Crop Science \& Technology. 5 (2): 1-8

Adhikari, B. N., Joshi, B. P., Shrestha, J., \& Bhatta, N. R. (2018). Genetic variability, heritability, genetic advance and correlation among yield and yield components of rice (Oryza sativa L.). Journal of Agriculture and Natural Resources, 1(1), 149-160.

Agricultural statistics. (2018). Pocket book on Agricultural statistics, ministry of Agricultural Government of India.

Barik, Jijnasa., Kumar, Vajinder., Pande, Debarata. (2020). Assessment of Variation in Morpho-Physiological Traits and Genetic Diversity in Relation to Submergence Tolerance of Five Indigenous Lowland Rice Landraces. Rice Science. 27(1): 32-43

Bhandari, K., Poudel, A., Sharma, S (2019). Genetic Variability, Correlation and Path Analysis of Rice in Rainfed Condition. Russian Journal of Agricultural Sciences, 8(92): 274-278 
Dhuri, S.Y., Bhati, P.K. and Saroj, S.K. (2014).Studies On Genetic Variability For Yield And Quality Characters In Rice (Oryza sativa L.) Under Integrated Fertilizer Management.The Bioscan.9(2): 745-748.

Directorate of Rice Research, Annual Report (2018-19), Rajendranagar, Hyderabad, 3: 133-135.

Falconer, D.S. (1960). Introduction to quantitative genetics Edinburgen and London:Aliver and Bodyd.

FAO (2016). Food and Agricultural organization. Online Interactive Database on Agriculture.

FAOSTAT.www.fao.org.

Fisher, R.A., Yates, F. (1967).Statistical tables for biological, Agricultural and medical Research, Congress Group Limited, London.

Ganapati, R K., Rasul, M G., Mian, M A K., Sarker, U., (2014) Genetic Variability and Character Association of T-Aman Rice (Oryza sativa L). International Journal of Plant Biology \& Research, 2(2): 1013

Gaurav Kumar, D. K., Dwivedi, Harishankar, M., and Yadav, V.K. (2018) Correlation and path coefficient analysis of thirteen rice genotypes for grain yield and other yield attributing traits. Journal of Pharmacognosy and Photochemistry, 7(3): 3506-3510.

Ghimire. N.H., and Mahat P. H., (2019) Genetic Variability and Correlation Coefficients of Major Traits in Cold Tolerance Rice (Oriza sativa L.) Under Mountain Environment of Nepal. International Journal of Applied Sciences Biotechnology, Vol 7(4): 445452

Girma BT., Kitil MA., Banje DG., Biru HM., Serbessa TB (2018) Genetic Variability Study of Yield and Yield Related Traits in Rice (Oryza sativa L.) Genotypes. Adv Crop Sci Tech 6
(4): 381-389.

Harsha., Deo, Indra., Kumar, Sudhir and Talha, Mohammed. 2017. Assessment of Genetic Variability and IenterCharacter Association Syudies in Rice Genotypes (Oryza sativa L.) International Journal of Current Microbiology and Applied Sciences. 6(10); 2865-2869.

Hussain, Md.Shakhawat., Monshi, Fakrul Islam., Tabassum, Raheenuma.(2017). Assesment of genetic variability of some exotic hybrid varieties of rice (Oryza sativa L.), Journal of plant science 12(1):22-29,2017.

IIRR, (2015).Indian Institute of Rice Research, Annual report Islam. M.J., Raffi. S.A., Hossain. M.A. and Hasan.A.K. (2015).Anaalysis of genetic variability, heritability and genetic advance for yield and yield associated traits in some promising advanced lines of rice(Oryza sativa L.). Progressive Agriculture.26 (1): 26-31.

Jambhulka, N.N. and Bose, L.K. (2014).Genetic variability and association of yield attributing traits with grain yield in upland rice. Genetika.46 (3): 831-838.

Kalyan, B., Radha Krishna, K.V., andSubbaRao, L.V.(2017)Correlation Coefficient Analysis for Yield and its Components in Rice (Oryza sativa L.) Genotypes. International Journal of Current Microbiology and Applied Sciences.6(7): 2425-2430.

Karthika, Gunasekaran., Rajeswari, S., Robin.S., Govintharaj. P., Vishnu, V. N., and Balaji, A. P.(2017) Assessment of genetic variability, correlation and path coefficient analysis for morphological and quality traits in rice (Oryza sativa L.).Agricultural Science Digest.,37(4): 251-256.

Kumar, A., Kumar, H., Guta, V., Khosta, G 
and Shrma, P.K. (2011). Correlation and path coefficient analysis for yield and yield component traits in rice (Oryza sativa L.). Agriulturec. Science. Digest., 31 (4): 275 - 279.

Maurya,B.K.,Singh,P.K.,Verma,O,P. and Mandal, D.K.(2017). Genetic Variability and Divergence analysis in Rice (Oryza sativa L.) International Journal of Current Microbiology and Applied Sciences.6(10); 2865-2869.

Nandan, R. and Shravan, K.S. (2018). Character association and path analysis in rice (Oryza sativa L.) genotypes. Agricultural Science Digest, 37 (3): 1012-1017.

Nuruzzaman, M., Hasan, L., Shamsun, N.B. and Huda, M.H. (2017). Correlation and path coefficient analysis of yield components in NERICA mutant rice lines under rained conditions. Journal of Experimental Agriculture International, 16 (1): 18.

Panse, V.G and Sukhatne, P.V.(1961). Statistical methods for agricultural workers 2nd Edn ICAR New Delhi P: 361.

Saha, S R., Hassan. L., Haque, A., Islam, M (2019) Genetic variability, heritability, correlation and path analyses of yield components in traditional rice (Oryza sativa L.) landraces. Journal
Bangladesh Agricultural University. 17(1): 26-32

Sala, M and Shanthi, P (2016). Variability, heritability and Genetic Advance studies in F2 population of rice (Oryza sativa L.). Internatinal Journal of Forestry and Crop Improvement, 7(1): 57-60

Sarker, M., Hassan, L., Rashid, M., Islam, S. N., (2019). Estimation of Variability, Heritability and Genetic Advance of Exotic Rice (Oryza sativa L.) Breeding Lines for Short Growth Development. Journal of Experimental Agriculture International. 32(3): 1-8

Singh, S.K., Singh, C.M and Lal, G.M. (2011).Assessment of genetic variability for yield and its component characters in rice (Oryza sativa L.). Research in Plant Biology, 1(4): 7376.

Tiwari, N. D., Tripathi, S. R., Tripathi, M. $\mathrm{P}(2019)$ Genetic Variability and Correlation Coefficients of Major Traits in Early Maturing Rice under Rainfed Lowland Environments of Nepal. Advances in Agriculture.

Vanisree, S., Swapna, K., Raju, C.H.S, Raju, C.S and Sreedhar, M. (2013). Genetic variability and selection criteria in rice. Journal of Biological and Scientific Opinion. 1 (4): 341-346.

\section{How to cite this article:}

Kritika Williams, Aman Mishra, Akansha Verma, BG. Suresh and Lavanya, G. R. 2021. Genetic Variability and Correlation Studies for Yield and Yield Related Traits in Rice (Oryza sativa L.) Genotypes. Int.J.Curr.Microbiol.App.Sci. 10(01): 752-764. doi: https://doi.org/10.20546/ijcmas.2021.1001.093 